\title{
A Surprising Photoactivity of Blue Fluorescent Protein TagBFP Allows for Super-Resolution Microscopy
}

\author{
DOI: $10.17691 / \mathrm{stm} 2018.10 .1 .04$
}

Received October 24, 2017

N.V. Klementieva, PhD, Junior Researcher, Laboratory of Super-Resolution Microscopy and Gene Technologies, Institute of Biomedical Technologies"; K.A. Lukyanov, DSc, Head of Biophotonics Laboratory2; Head of Fluorescent Bioimaging Laboratory, Institute of Biomedical Technologies';

D.A. Gorbachev, Junior Researcher, Biophotonics Laboratory2; PhD Student, Faculty of Biology3:

D.M. Chudakov, DSc, Head of the Laboratory of Immunosequencing Methods'; Research Group Leader ${ }^{4}$;

E.V. Zagaynova, MD, DSc, Professor of Russian Academy of Sciences,

Director of the Institute of Biomedical Technologies";

A.S. Mishin, PhD, Senior Researcher, Biophotonics Laboratory2; Head of the Laboratory

of Super-Resolution Microscopy and Gene Technologies, Institute of Biomedical Technologies ${ }^{1}$

${ }^{1}$ Privolzhsky Research Medical University, 10/1 Minin and Pozharsky Square, Nizhny Novgorod, 603005, Russia;

${ }^{2}$ Shemyakin-Ovchinnikov Institute of Bioorganic Chemistry, Russian Academy of Sciences,

16/10 Miklukho-Maklaya St., Moscow, 117997, Russia;

${ }^{3}$ Lomonosov Moscow State University, 1 Leninskie Gory, Moscow, 119991, Russia;

${ }^{4}$ Central European Institute of Technology, Kamenice 753/5, Brno, 60177, Czech Republic

The aim of this research was to study photobehavior of a popular blue fluorescent protein TagBFP and apply this marker for superresolution microscopy.

Materials and Methods. Photoactivation of TagBFP was examined both in protein solution in vitro and in living cells. Subdiffraction imaging was performed using total internal reflection fluorescence microscopy followed by super-resolution radial fluctuations or singlemolecule localization analysis.

Results. We show that TagBFP exhibits blinking behavior upon $405 \mathrm{~nm}$ light illumination. Moreover, photoactivation to red-emitting state is occurring in the conditions typically used for TagBFP imaging. The red (photoactivated) form of TagBFP possesses spectral properties similar to TagRFP - a close homologue of TagBFP. We show that both blinking and photoactivation of TagBFP can be utilized for super-resolution imaging. We conclude that photoactivation of TagBFP to red-emitting form should be taken into account in the design of multi-channel imaging experiments involving high-power or prolonged UV illumination.

Key words: TagBFP; photoactivation; blue-to-red photoconversion; blinking; super-resolution microscopy.

How to cite: Klementieva N.V., Lukyanov K.A., Gorbachev D.A., Chudakov D.M., Zagaynova E.V., Mishin A.S. A surprising photoactivity of blue fluorescent protein TagBFP allows for super-resolution microscopy. Sovremennye tehnologii v medicine 2018; 10(1): 35, https://doi. org/10.17691/stm2018.10.1.04

\section{Introduction}

Fully genetically encoded labels - fluorescent proteins of the green fluorescent protein (GFP) family enable realtime visualization of various structures and events in live cells [1]. They provide a rich information in multicolor imaging regime that makes it possible to establish a relationship between different cellular targets in space and time. Unlike other parts of the visible spectrum, the palette of widely used blue fluorescent proteins is limited to only a few bright and photostable monomeric proteins, namely Azurite, EBFP2, and TagBFP [2-4]. Therefore, these proteins are often included as a blue tag in multicolor imaging experiments.

Regular fluorescence microscopy provides the maximum resolution of $200-350 \mathrm{~nm}$ that makes many important fine features of a target structure hidden. This basic problem has been recently solved by superresolution fluorescence microscopy techniques, which ensure subdiffraction resolution down to $10-20 \mathrm{~nm}$ [5]. Subdiffraction imaging methods based on single-molecule localization microscopy provide the highest resolution but require special fluorescent labels [6]. Only some types of these probes are suitable for fast imaging of live cells [7].

Corresponding author: Alexander S. Mishin, e-mail: mishin@ibch.ru 
We have previously reported that TagRFP and several closely related red fluorescent proteins exhibit fast reversible photoconversion between dark and bright states, which could be utilized for super-resolution imaging [8]. We, therefore, sought to assess the photoconversion in a closely related fluorescent protein TagBFP [4], which is a blue-emitting variant of TagRFP.

The aim of this research was to study photobehavior of TagBFP and apply this genetically encoded fluorescent marker for super-resolution microscopy.

\section{Materials and Methods}

Plasmids and protein purification. We used actininTagBFP and Lifeact-TagBFP plasmids from Evrogen (Russia). TagBFP in $\mathrm{pQE}-30$ vector with $6 \mathrm{His}$-tag at the $\mathrm{N}$-terminus was used for protein expression. After sonication of the bacterial suspension, the protein was purified from soluble fraction using TALON metal-affinity resin (Clontech, USA) according to the manufacturer's protocol. The protein solution (in phosphate-buffered saline) was illuminated with $405 \mathrm{~nm}$ light $\left(\sim 1 \mathrm{~W} / \mathrm{cm}^{2}\right.$ from X-Cite XLED1 light source) (Excelitas Technologies, USA) for $30 \mathrm{~min}$. Absorbance and fluorescence spectra of TagBFP were recorded using a Cary 100 spectrophotometer (Varian, USA) and a Cary Eclipse fluorescence spectrophotometer (Varian, USA).

Cell culture. HeLa (human cervical carcinoma) cells and NIH 3T3 (mouse embryo fibroblasts) cells were cultured in DMEM (PanEco, Russia) supplemented with $10 \%$ fetal bovine serum (HyClone, USA), $2 \mathrm{mM}$ L-glutamine (PanEco, Russia), and antibiotics (50 U/ml penicillin plus $50 \mu \mathrm{g} / \mathrm{ml}$ streptomycin) (PanEco, Russia) at $37^{\circ} \mathrm{C}$ in humidified atmosphere with $5 \% \mathrm{CO}_{2}$. Cells were passaged every 3 days.

Total internal reflection fluorescence (TIRF) microscopy and data analysis. TIRF imaging was carried out on an Eclipse Ti N-STORM microscope (Nikon, Japan) with NIS-Elements 4.13.01 Software and 100x oil-immersion TIRF objective (Apo TIRF/1.49; Nikon, Japan). Time series were acquired with Andor iXon3 DU-897 camera (Andor, UK), EM gain of 200 or 296 , and pre-amplifier gain of $5.1 \times$ or $2.4 \times$, with a pixel size of $160 \mathrm{~nm}$. The samples were illuminated using 405 or $561 \mathrm{~nm}$ laser lines. Data processing and image reconstruction for super-resolution radial fluctuations (SRRF) were performed as described [9] with 100500 raw frames. Single-molecule localization analysis was performed with ThunderSTORM [10] software.

\section{Results and Discussion}

Fast reversible photoconversion of TagBFP. We found that TagBFP, similarly to TagRFP, exhibits robust frame-to-frame pixel intensity fluctuations with exposures between 10 to $100 \mathrm{~ms}$ and illumination intensities as low as $0.1 \mathrm{~W} / \mathrm{cm}^{2}$ of $405 \mathrm{~nm}$ light illumination. We revealed that further increase in illumination intensity does not increase the variance-to-mean ratio of fluctuation. We then processed the raw data with recently developed SRRF algorithm [9], which took the advantage of temporally correlated fluctuations resulting from multiple blinking events of overlapping fluorophores. A representative image demonstrating an improvement in spatial resolution compared to TIRF microscopy is shown in Figure 1.
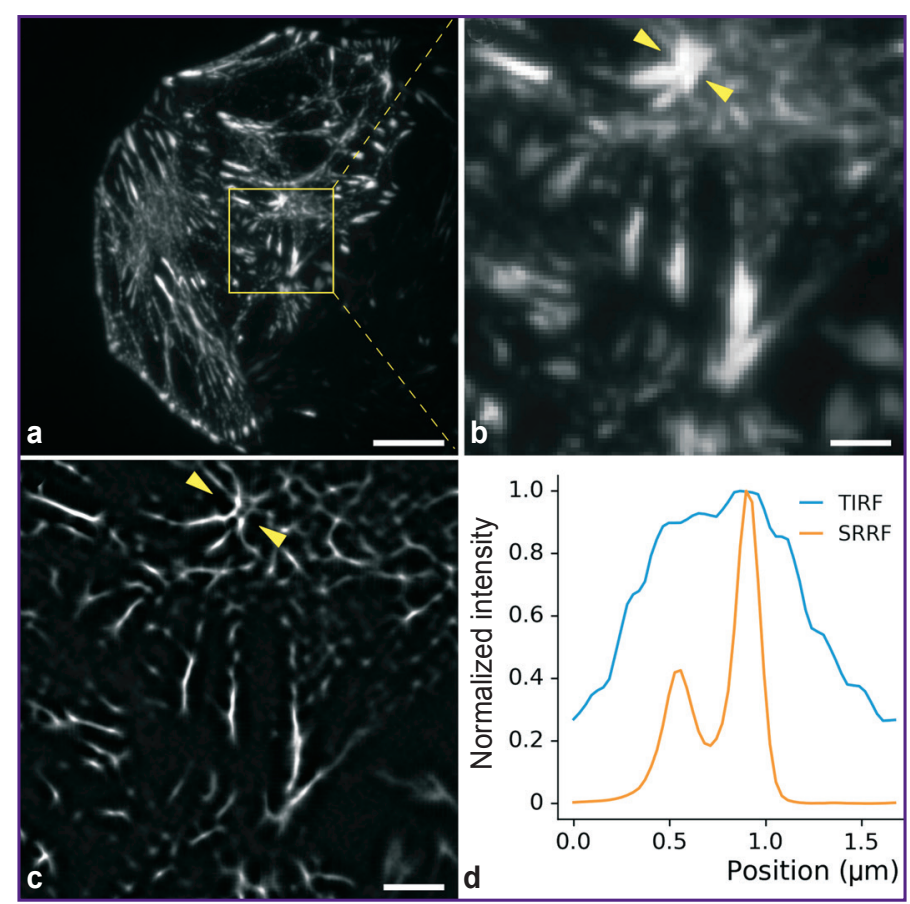

Figure 1. Super-resolution imaging with TagBFP Live HeLa cells labeled with actinin-TagBFP. Image reconstruction by SRRF (super-resolution radial fluctuations) algorithm from 300 consequent frames at $50 \mathrm{~ms}$ camera exposure time, $405 \mathrm{~nm}$ light excitation at $0.1 \mathrm{~W} / \mathrm{cm}^{2}$. (a) TIRF (total internal reflection fluorescence) image, scale bar $10 \mu \mathrm{m}$; (b) enlarged fragment from panel (a), scale bar $2 \mu \mathrm{m}$; (c) SRRF image of the same region, scale bar $2 \mu \mathrm{m}$; (d) intensity profiles between yellow arrows on panels $b-$ blue lines and $c-$ orange lines 

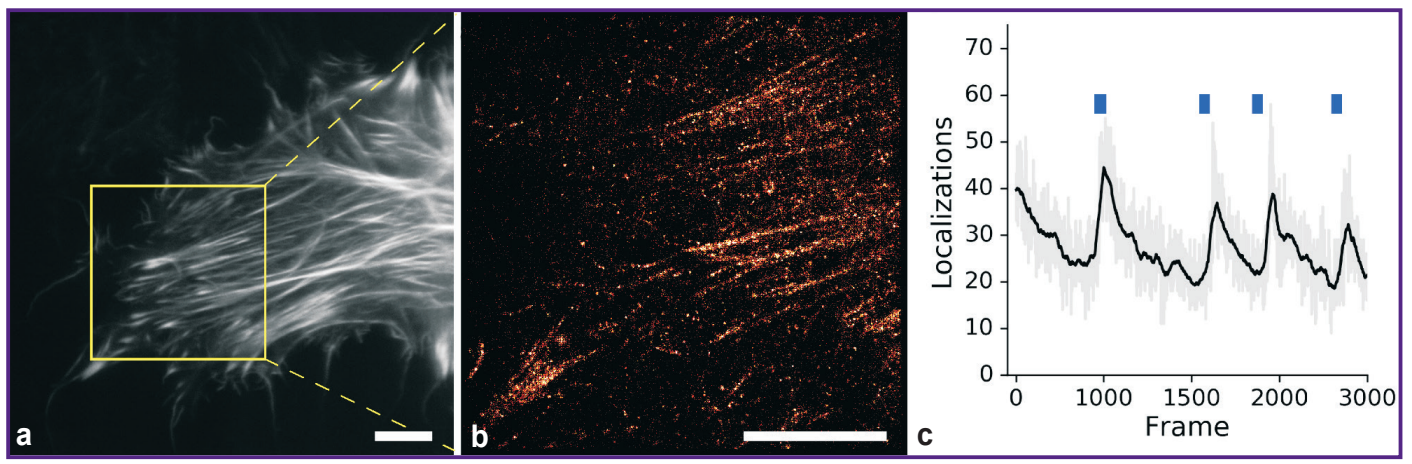

Figure 2. Photoactivation of TagBFP to red-emitting form

Live NIH 3T3 cells labeled with Lifeact-TagBFP. (a) TIRF (total internal reflection fluorescence) image, $405 \mathrm{~nm}$ excitation, scale bar $10 \mu \mathrm{m}$; (b) super-resolution image, $561 \mathrm{~nm}$ excitation $\left(60 \mathrm{~W} / \mathrm{cm}^{2}\right)$, the total of $\sim 110,000$ localizations are shown, scale bar $10 \mu \mathrm{m}$; (c) dependence of the number of single-molecule localizations on the $405-\mathrm{nm}$ photoactivation (blue squares, $\sim 1$-s pulse of $0.3 \mathrm{~W} / \mathrm{cm}^{2}$ ). Smoothed (black) line is added to aid the visualization

In cellulo photoactivation of TagBFP. The TagBFPlike chromophore is believed to be a precursor of DsRed-like chromophores of red fluorescent proteins, and also represents the dark states of some irreversibly photoactivated fluorescent proteins [11, 12]. To test this idea, we illuminated TagBFP-labeled live cells with 405 laser of varying intensity, followed by imaging with $561 \mathrm{~nm}$ excitation. The increase in red fluorescence was clearly visible right after $\sim 1$-s pulses of 405 laser $\left(0.3-1.0 \mathrm{~W} / \mathrm{cm}^{2}\right)$. The photoconversion efficiency of TagBFP was at least an order of magnitude lower than the one of Dendra2 (not shown) under similar conditions. Nonetheless, we localized more than 100,000 singlemolecule bursts of red fluorescence in a $500 \mu \mathrm{m}^{2}$ region of the cell in less than $2.5 \mathrm{~min}$ with a total of $5 \mathrm{~s}$ of $0.3 \mathrm{~W} / \mathrm{cm}^{2} 405-\mathrm{nm}$ laser illumination (Figure 2).

In vitro photoactivation of TagBFP. We detected that purified TagBFP in a solution shows a sharp increase in $\sim 555 \mathrm{~nm}$ absorbance after 30-min illumination with high-power 405-nm light-emitting diode (Figure 3). The absorbance of the photoproduct $(555 \mathrm{~nm})$ coincides almost perfectly with published TagRFP absorbance spectra. Surprisingly, we did not observe a significant increase in the fluorescence from the same sample (data not shown). Presumably, the emerging red form appears initially in the dark state. Therefore, the photoconversion of TagBFP demonstrated for the first time in current work may have been overlooked by previous studies.

\section{Conclusion}

The photoconversion of TagBFP into red-emitting form is a particularly disturbing phenomenon for the design of super-resolution experiments. Specifically, the red-emitting form of TagBFP could be easily confused with red-emitting forms of other photoactivated proteins, such as Dendra2. In many high-resolution experimental setups, the optical components are specifically designed to maximize photon count of detected localization, with

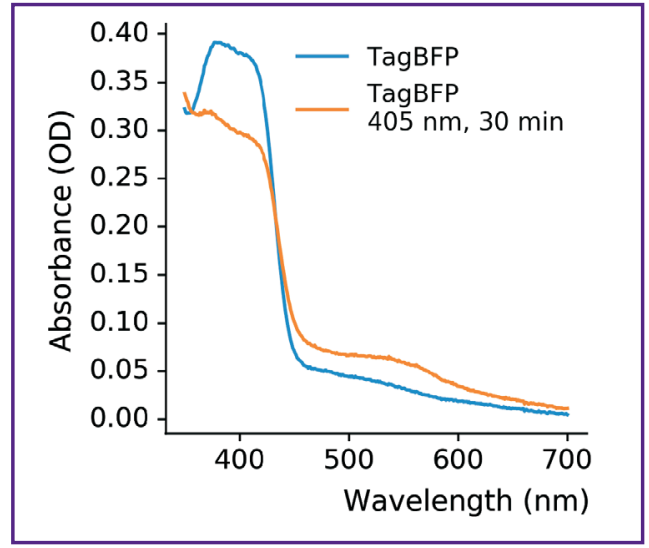

Figure 3. In vitro photoactivation of TagBFP

Absorbance spectra of a TagBFP solution (phosphate-buffered saline, $\mathrm{pH}$ 7.4) before (blue lines) and after (orange lines) photoactivation with high-power 405-nm light-emitting diode

little or no spectral filtering (for instance, filter-cubes with multiband dichroic mirrors, without any suppression filters). While we found the efficiency of blue-to-red photoactivation of TagBFP insufficient for dedicated single-molecule localization imaging, the possible artifacts should be considered with any combination of TagBFP with UV-induced photoactivatable fluorescent proteins, such as Dendra2, mEos3.2, PA-GFP, PA-TagRFP, PAmCherry, etc. In contrast, the blinking behavior of TagBFP allowing for super-resolution microscopy gives an additional fluorescent tool for researchers.

Acknowledgments. Experiments were partially carried out using the equipment provided by the IBCh core facility (CKP IBCh, supported by Russian Ministry of Education and Science, grant RFMEFI62117X0018). NVK was supported by the Russian Presidential Scholarship (СП-4194.2016.4). 
Conflict of Interests. The authors declare no conflict of interests.

\section{References}

1. Mishin A.S., Belousov V.V., Solntsev K.M., Lukyanov K.A Novel uses of fluorescent proteins. Curr Opin Chem Biol 2015; 27: 1-9, https://doi.org/10.1016/j.cbpa.2015.05.002.

2. Mena M.A., Treynor T.P., Mayo S.L., Daugherty P.S. Blue fluorescent proteins with enhanced brightness and photostability from a structurally targeted library. Nat Biotechnol 2006; 24(12): 1569-1571, https://doi.org/10.1038/nbt1264.

3. Ai H., Shaner N.C., Cheng Z., Tsien R.Y., Campbell R.E. Exploration of new chromophore structures leads to the identification of improved blue fluorescent proteins. Biochemistry 2007; 46(20): 5904-5910, https://doi. org/10.1021/bi700199g.

4. Subach O.M., Gundorov I.S., Yoshimura M., Subach F.V., Zhang J., Grüenwald D., Souslova E.A., Chudakov D.M., Verkhusha V.V. Conversion of red fluorescent protein into a bright blue probe. Chem Biol 2008; 15(10): 11161124, https://doi.org/10.1016/j.chembiol.2008.08.006.

5. Klementieva N.V., Zagaynova E.V., Lukyanov K.A., Mishin A.S. The principles of super-resolution fluorescence microscopy (review). Sovremennye tehnologii $v$ medicine 2016; 8(2): 130-140, https://doi.org/10.17691/stm2016.8.2.17.

6. Wegel E., Göhler A., Lagerholm B.C., Wainman A., Uphoff S., Kaufmann R., Dobbie I.M. Imaging cellular structures in super-resolution with SIM, STED and localisation microscopy: a practical comparison. Sci Rep 2016; 6: 27290 https://doi.org/10.1038/srep27290.

7. Klementieva N.V., Bozhanova N.G., Zagaynova E.V., Lukyanov K.A., Mishin A.S. Fluorophores for single-molecule localization microscopy. Russ J Bioorganic Chem 2017; 43(3): 227-234, https://doi.org/10.1134/s1068162017030074.

8. Klementieva N.V., Pavlikov A.I., Moiseev A.A., Bozhanova N.G., Mishina N.M., Lukyanov S.A., Zagaynova E.V., Lukyanov K.A., Mishin A.S. Intrinsic blinking of red fluorescent proteins for super-resolution microscopy. Chem Commun 2017; 53(5): 949-951, https://doi.org/10.1039/c6cc09200d.

9. Gustafsson N., Culley S., Ashdown G., Owen D.M., Pereira P.M., Henriques R. Fast live-cell conventional fluorophore nanoscopy with ImageJ through super-resolution radial fluctuations. Nat Commun 2016; 7: 12471, https://doi. org/10.1038/ncomms12471.

10. Ovesný M., Křížek P., Borkovec J., Švindrych Z., Hagen G.M. ThunderSTORM: a comprehensive ImageJ plugin for PALM and STORM data analysis and super-resolution imaging. Bioinformatics 2014; 30(16): 2389-2390, https://doi. org/10.1093/bioinformatics/btu202.

11. Shcherbakova D.M., Verkhusha V.V. Chromophore chemistry of fluorescent proteins controlled by light. Curr Opin Chem Biol 2014; 20: 60-68, https://doi.org/10.1016/j. cbpa.2014.04.010.

12. Subach O.M., Cranfill P.J., Davidson M.W., Verkhusha V.V. An enhanced monomeric blue fluorescent protein with the high chemical stability of the chromophore. PLoS One 2011; 6(12): e28674, https://doi.org/10.1371/journal. pone.0028674. 